\title{
ON PROJECTIVELY FLAT HERMITIAN MANIFOLDS
}

\author{
JUN LI, SHING-TUNG YAU†, AND FANGYANG ZHENG
}

Let $\left(M^{n}, g\right)$ be a $n$-dimensional compact hermitian manifold, with $n \geq 2$. $(M, g)$ will be called projectively flat, if its curvature matrix is of the form $\Theta=\alpha I_{n}$, where $\alpha$ is a $(1,1)$-form. Note that any metric conformal to $g$ would also be projectively flat. In $\S 1$, we shall classify such manifolds, and in $\S 2$, we will give an application which may be considered as a generalization to higher dimensions of the Bogomolov's Theorem on $\mathrm{VII}_{0}$ surfaces.

First of all, let us correct an error in our previous paper [L-Y-Z]. We found this error after the paper was in print. On page 220, the vanishing of $c_{1}^{2}$ and $c_{2}$ does not imply that the Hermitian-Einstein metric $h$ is flat, but only projectively flat, i.e., $\Theta=\alpha I_{2}$. So the argument there is incomplete. However, this gap can be easily fixed by applying the results of P. Gauduchon ([G]) and D. Fried $([\mathrm{F}])$. Start from the projectively flat compact hermitian surface $(S, h)$. By $[\mathrm{G}], h$ is locally conformally Kähler. That is, there exists a covering $\left\{U_{\alpha}\right\}$, and $f_{\alpha} \in \mathcal{C}^{\infty}\left(U_{\alpha}, R\right)$, such that each $e^{f_{\alpha}} g$ is a Kähler metric in $U_{\alpha}$. Note $e^{f_{\alpha}} g$ is also projectively flat, hence flat, and in $U_{\alpha} \cap U_{\beta}, f_{\alpha}-f_{\beta}$ is a constant. Therefore, $S$ is a complex similarity manifold. By Theorem 2 of $[\mathrm{F}]$, it is either covered by a complex 2 -torus or a Hopf surface. This completes the proof of Bogomolov's theorem. ${ }^{1}$

\section{PRoJeCtiVELY FLAT MANIFOLDS}

Now let us consider the projectively flat manifolds in general dimensions. First let us fix some notations. On a hermitian manifold $\left(M^{n}, g\right)$, let $e=$

$\nmid$ Research partially supported by the NSF grant DMS-92-06938.

$\ddagger$ Research partially supported by the NSF grant DMS-93-08239.

${ }^{1}$ This error was also recently pointed out to us by A. Teleman, who found another interesting way to correct it. 
$\left(e_{1}, \ldots, e_{n}\right)$ be a local unitary frame and $\varphi=\left(\varphi_{1}, \ldots, \varphi_{n}\right)$ its dual coframe. Let $\theta, \Theta$ the $n \times n$ matrices of connection and curvature under $e$, and $\tau=$ $\left(\tau_{1}, \ldots, \tau_{n}\right)$ the torsion forms under $e$. Each $\tau_{i}$ is a $(2,0)$-form. The structure equations and the first Bianchi identity are:

$$
d \varphi=\varphi \wedge \theta+\tau, \quad d \theta-\theta \wedge \theta=\Theta, \quad d \tau=\varphi \wedge \Theta-\tau \wedge \theta
$$

Write $\tau_{i}=\frac{1}{2} \sum_{j, k=1}^{n} T_{j k}^{i} \varphi_{j} \wedge \varphi_{k}$, where $T_{j k}^{i}=-T_{k j}^{i}$, and denote by $\omega=\varphi \wedge \varphi^{*}$ the Kähler form of $g$ (we omit the factor $\sqrt{-1}$ ).

Consider the Gauduchon torsion 1-form $\eta$ ([G]) defined by

$$
\eta=\frac{1}{n-1} \sum_{j, k=1}^{n} T_{j k}^{k} \varphi_{j}
$$

It is easy to check that $\partial\left(\omega^{n-1}\right)=(n-1) \eta \wedge \omega^{n-1}$, hence is uniquely determined and globally defined. First of all, one has:

Lemma 1. If $\Theta=\alpha I_{n}$, then $\bar{\partial} \eta=\alpha$.

Proof. Write the $(0,1)$ part of $\theta$ as $\theta_{i j}^{\prime \prime}=\sum_{l=1}^{n} A_{i j, \bar{l}} \bar{\varphi}_{l}$ and $\alpha=\sum_{i, j=1}^{n} \alpha_{i \bar{j}} \varphi_{i} \wedge \bar{\varphi}_{j}$. By the structure equation and the first Bianchi identity,

$$
\bar{\partial} \varphi=\varphi \wedge \theta^{\prime \prime} ; \quad \bar{\partial} \tau=\varphi \wedge \alpha-\tau \wedge \theta^{\prime \prime}
$$

Hence for each $i, j, k$, and $l$, one has:

$$
\nabla_{\bar{l}} T_{j k}^{i}=\delta_{i j} \alpha_{k \bar{l}}-\delta_{i k} \alpha_{j \bar{l}}+\sum_{r=1}^{n} T_{r k}^{i} A_{j r, \bar{l}}-\sum_{r=1}^{n} T_{r j}^{i} A_{k r, \bar{l}}-\sum_{r=1}^{n} T_{j k}^{r} A_{r i, \bar{l}}
$$

Therefore

$$
\sum_{k=1}^{n} \nabla_{\bar{l}} T_{j k}^{k}=-(n-1) \alpha_{j \bar{l}}+\sum_{r, k=1}^{n} T_{r k}^{k} A_{j r, \bar{l}}
$$

This leads to $\bar{\partial} \eta=\alpha$, and the lemma is proved.

Next let $\sigma=(\tau-\eta \wedge \varphi) \otimes{ }^{t} e$. Then one has $\bar{\partial} \sigma=(\alpha-\bar{\partial} \eta) \varphi \otimes^{t} e=0$. So $\sigma$ is a holomorphic section of $E=\Omega_{M} \otimes \Omega_{M} \otimes T_{M}$, where $T_{M}, \Omega_{M}$ denotes the holomorphic tangent and cotangent bundle of $M$. Let $h$ be the hermitian metric on $E$ induced from $g$ on $T_{M}$. Fix a point $x \in M$, choose holomorphic 
frame $v_{1}, \ldots, v_{N}$ of $E$ near $x$, so that at $x, h_{i \bar{j}}=\delta_{i j}, d h_{i \bar{j}}=0$. Write $\sigma=$ $\sum_{i=1}^{N} \sigma_{i} v_{i}$, then at $x$ :

$$
\partial \bar{\partial}\|\sigma\|^{2}=\sum_{i=1}^{N} \partial \sigma_{i} \wedge \overline{\partial \sigma_{i}}-\Theta_{\sigma \bar{\sigma}}(h) \geq\|\sigma\|^{2} \alpha
$$

Here we used the fact that $\Theta(h)=-\alpha I_{N}$. Note that if $\partial \bar{\partial} \omega^{n-1}=0$, then $\bar{\partial} \eta \wedge \omega^{n-1}=\eta \wedge \bar{\eta} \wedge \omega^{n-1}$. When $M$ is compact,

$$
0=\int \partial \bar{\partial}\|\sigma\|^{2} \wedge \omega^{n-1} \geq \int\|\sigma\|^{2} \eta \wedge \bar{\eta} \wedge \omega^{n-1}
$$

therefore we have

Lemma 2. If $M$ is compact, $\Theta=\alpha I_{n}$, and $\partial \bar{\partial} \omega^{n-1}=0$, then either $\eta=0$ or $\tau=\eta \wedge \varphi$.

When $\eta=0,((M, g)$ is called balanced, $)$ the first Ricci form $r$ equals to the third Ricci form $s$ :

$$
r_{i \bar{j}}-s_{i \bar{j}}=\sum_{k=1}^{n} R_{k \bar{k} i \bar{j}}-R_{i \bar{k} k \bar{j}}=\nabla_{\bar{j}} \sum_{k=1}^{n} T_{i k}^{k}=0
$$

But for projectively flat metric, $r-s=(n-1) \alpha$, so $\eta=0$ implies $\Theta=0$ in this case.

While when $\tau=\eta \wedge \varphi, \partial \omega=\tau \wedge \varphi^{*}=\eta \wedge \omega$. Hence $\partial \eta \wedge \omega=0$. When $n \geq 3$, this gives $\partial \eta=0$, while when $n=2$, since $\bar{\partial} \eta=\alpha$ is closed, $0=\int \partial \eta \wedge \overline{\partial \eta}$ implies $\partial \eta=0$. Therefore $d(\eta+\bar{\eta})=\alpha+\bar{\alpha}=0$, so locally $g$ will be conformal to some Kähler metric, which is necessarily flat. So $M$ is a complex similarity manifold. By $[\mathrm{F}],(M, g)$ is a finite undercover of either a flat complex torus, or a Hopf manifold of the form $\left(C^{n} \backslash 0\right) / Z \phi$, where $\phi(z)=a z A$ is a complex expansion: $A \in U(n), a>1$ and $z=\left(z_{1}, \ldots, z_{n}\right)$.

In conclusion, one has:

Theorem 1. Let $\left(M^{n}, g\right)$ be a compact projectively flat hermitian manifold, and suppose its Kähler form $\omega$ satisfies $\partial \bar{\partial} \omega^{n-1}=0$. Then either $(M, g)$ is flat and balanced $\left(d \omega^{n-1}=0\right)$, or $M$ is a finite undercover of a quotient $C^{n} \backslash 0 / Z \phi$ with $\phi$ a complex expansion. 
Note that by [G1], for any compact hermitian manifold $(M, g)$, there exists an unique (up to homothety) metric $h$ in the conformal class of $g$ such that $\partial \bar{\partial} \omega_{h}^{n-1}=0$; and if $g$ is projectively flat, so is $h$. Therefore, any projectively flat metric on $M$ is conformal to one of the metrics in Theorem 1.

For compact hermitian flat manifold $(M, g)$, the torsion tensor is parallel. So the first Bianchi identity gives exactly the Poisson identities, and the universal covering space is a complex Lie group $G$ equipped with an left invariant flat metric. That is, $M=\Gamma \backslash G$, where $\Gamma \subset G \cdot C$ is a discrete subgroup of the semidirect product of $G$ with a compact subgroup $C \subset \operatorname{Aut}(G)$. See [Go] or [K-T] for example.

As a byproduct, we get the following

Corllary 2. Any compact hermitian flat manifold $\left(M^{n}, g\right)$ is balanced, i.e., $d \omega_{g}^{n-1}=0$.

This is because we can first conformally deform $g$ to get a balanced and projectively flat metric $h=e^{f} g$. Since any connected Lie group $G$ is either a $K(\pi, 1)$ or has $\pi_{3}(G) \neq 0$, so $G$ can not be homotopic to $S^{2 n-1}$ if $n \geq 3$; while when $n=2$, there are only two simply-connected complex Lie groups, both biholomorphic to $C^{2}$, so $M$ can not be Hopf. Therefore, by Theorem 1 , we know $h$ is again flat, so $f$ is pluriharmonic, hence a constant, and $g$ is balanced.

In particular, for $n=2$, we get the well-known fact that any compact hermitian flat surface has to be Kähler, namely, a complex 2-torus or a hyperelliptic surface.

\section{AN COROLLARY}

By the proof of $[\mathrm{L}-\mathrm{Y}-\mathrm{Z}]$, the theorem of Bogomolov on $\mathrm{VII}_{0}$ surfaces $([\mathrm{B}]$, [B1]) can now be stated in a slightly more general way, namely, if $M^{2}$ is a compact complex surface with stable tangent bundle $T_{M}$ (with respect to a hermitian metric) and with $c_{1}^{2}=c_{2}=0$, then $M$ must be either flat or similarity Hopf.

In this section, we want to generalize this into higher dimensions by apply Theorem 1 in $\S 1$. First let us recall the definition of refined Chern classes by 
Bott and Chern ([B-C]). Suppose $E$ is a holomorphic vector bundle over a compact complex manifold $M^{n}$. Then for any two hermitian metrics $h, h^{\prime}$ on $E$, there always exists smooth functions $f_{k}$ such that $\sqrt{-1} \partial \bar{\partial} f_{k}=C_{k}(h)-$ $C_{k}\left(h^{\prime}\right)$, where $C_{k}$ denotes the Chern forms. Hence the Chern forms define the refined Chern classes $\hat{c}_{k}(E)$ in $\hat{H}^{k, k}(M)=\operatorname{Ker}(d) \cap A^{k, k} / \operatorname{Im}(\sqrt{-1} \partial \bar{\partial})$. Here $A^{k, k}$ is the space of all smooth real $(k, k)$ forms on $M$.

Next, let us recall the definition of astheno-Kähler from [J-Y]. A hermitian metric $g$ on a compact complex $n$-manifold $M$ is called astheno-Kähler, if its metric form $\omega_{g}$ satisfies $\partial \bar{\partial} \omega_{g}^{n-2}=0$.

Any product manifold of curves and surfaces is astheno-Kähler. However, it would be more intersting to construct some "non-trivial" examples.

A necessary condition for the existence of such metrics is that, any semipositive $(2,2)$ current can not be $\partial \bar{\partial}$-exact (unless it is trivial). Note that for $n=3$, this is also a sufficient condition. (More generally, on a compact complex manifold, the non-existence of (non-trivial) $\partial \bar{\partial}$-exact positive $(n-1, n-1)$ current (acting on $(1,1)$ forms) always implies the existence of a hermitian metric $g$ with $\partial \bar{\partial} \omega_{g}=0$. Following the work of Harvey and Lawson ([H-L]), this is not hard to show.)

In particular, any global holomorphic 1-form $\varphi$ on $M$ must be closed, as $\partial \bar{\partial}(\varphi \wedge \bar{\varphi})=-\partial \varphi \wedge \overline{\partial \varphi} \geq 0$. So, for example, a compact complex parallelizable manifold $M$ (i.e., $T_{M}$ holomorphicly trivial) can not be astheno-Kähler unless it is a complex torus. However, we believe that this definition has its potential in the future study of the non-Kähler geometry.

Now if we start off with a compact astheno-Kähler manifold $\left(M^{n}, g\right)$. Let $E$ be a $g$-polystable holomorphic vector bundle of rank $r$ on $M$ (i.e., $E$ is the direct sum of $g$-stable bundles with the same $g$-slope ( $g$-degree divided by rank)). Then by [L-Y] (when $n=2$, also by Buchdahl [Bu]), $E$ admits a hermitian metric $h$ which is $g$-Einstein: $\operatorname{tr}_{g}\left(\Theta_{h}\right)=\mu I_{r}$ for some constant $\mu$. By the Lübke-Kobayashi inequality, $\left(C_{1}^{2}(E, h)-\frac{2 r}{r-1} C_{2}(E, h)\right) \wedge \omega_{g}^{n-2} \leq 0$ pointwisely. So if $\hat{c}_{1}^{2}(E)-\frac{2 r}{r-1} \hat{c}_{2}(E)=0$ in $\hat{H}^{2,2}(M)$ (or $\geq 0$ in the obvious sense), then $(E, h)$ is projectively flat: $\Theta_{h}=\alpha I_{r}$. In particular, when $E=T_{M}$, by Theorem1, we get the following: 
Corllary 3. Let $\left(M^{n}, g\right)$ be a compact hermitian manifold which is asthenokähler (i.e., $\partial \bar{\partial} \omega_{g}^{n-2}=0$ ). Suppose that $T_{M}$ is g-polystable and the refined Chern classes satisfy $\hat{c}_{1}^{2}=\hat{c}_{2}=0$ in $\hat{H}^{2,2}(M)$. Then either $M$ is similarity Hopf, or it admits a flat hermitian metric $h$.

Obviously the condition on the refined Chern classes can be replaced by $\hat{c}_{1}^{2}-$ $\frac{2 n}{n-1} \hat{c}_{2} \geq 0$ in the sense that it can be represented by a pointwisely nonnegative $(2,2)$ form, or that its product with any $[\Omega]$ is nonnegative, for any $\partial \bar{\partial}$-closed nonnegative $(n-2, n-2)$ form $\Omega$ on $M$.

We also conjecture that the non-Kähler flat manifolds or similarity Hopf manifolds of dimension $\geq 3$ do not admit astheno-Kähler metrics. This is true in some special cases, but at this moment we are unable to prove it in general. After this, the conclusion of Corollary 3 could be replaced by: " $M$ is covered by a complex torus".

Acknowledgement. We would like to thank the referee of the article for several helpful suggestions.

\section{REFERENCES}

[B] Bogomolov, F.A., Classification of surfaces of class VII $I_{0}$ with $b_{2}=0$, Math. USSR-Izv. 10 (1976), 255-269.

[B1] Bogomolov, F.A., Surfaces of class VII $I_{0}$ and affine geometry, Math. USSRIzv. 21 (1983), 31-73.

[Bu] Buchdahl, N.P., Hermitian-Einstein connections and stable vector bundles over compact complex surfaces, Math. Ann. 280 (1988), 625-648.

[B-C] Bott, R. and Chern, S.S., Hermitian vector bundles and the equidistribution of zeroes of their holomorphic sections, Acta Math., 114 (1965), 71-112.

[F] Fried, D., Closed similarity manifolds, Comm. Math. Helv. 55 (1980), 576582 .

[G] Gauduchon, P., La 1-forme de torsion d'une variété hermitienne, Math. Ann. 267 (1984), 495-518.

[G1] Gauduchon, P., Le théorème de l'excentricité nulle, C.R. Acad. Sci. Paris 258 (1953), 387-390.

[Go] Goldberg, S., Curvature and Topology, Academic Press, New York and London 1962.

[H-L] Harvey, R. and Lawson, H.B. Jr., An intrinsic characterization of Kähler manifolds, Invent. Math. 74 (1983), 169-198

[J-Y] Jost, J. and Yau, S.-T., A nonlinear elliptic system for maps from Hermitian to Riemannian manifolds and rigidity theorems in Hermitian geometry, to appear in Acta. Math.. 
[K-T] Kamber, F. and Tondeur, P., Flat Manifolds, Lecture Notes in Math. vol.67, Springer-Verlag, New York 1968.

[L-Y] Li, J. and Yau, S.-T., Hermitian-Yang-Mills connections on non-Kähler manifolds, in "Mathematical Aspects of String Theory", (ed. by S.T. Yau), World Sci. Publ. London, 1987, 560-573.

[L-Y-Z] Li, J., Yau, S.-T. and Zheng, F., A simple proof of Bogomolov's theorem on class $V I I_{0}$ surfaces with $b_{2}=0$, Illinois J. Math. 34 (1990), 217-220.

Stanford University, Stanford, U. S. A.

Harvard University, Cambridge, U. S. A.

Duke University, Durham, U. S. A.

RECEIVED JULY 12, 1993 